\title{
ESCLEROSE MÚLTIPLA
}

\section{ESTUDO DESCRITIVO DE SUAS FORMAS CLÍNICAS EM 302 CASOS}

\author{
MARCOS AURÉLIO MOREIRA*, EDUARDO FELIPE**, \\ MARIA FERNANDA MENDES**, CHARLES PETER TILBERY***
}

\begin{abstract}
RESUMO - A esclerose múltipla é uma das causas mais comuns de incapacidade neurológica crônica em adultos jovens. Avaliamos, através de estudo retrospectivo, características epidemiológicas, formas de apresentação, manifestações clínicas, evolução e o grau de incapacitação física da esclerose múltipla em 302 pacientes. A média da idade dos pacientes foi 37,7 anos e a relação entre os gêneros foi 3,13F:1M. A média da idade no início da doença foi 29,6 anos. Duzentos e oitenta e três pacientes eram brancos (94\%), 15 negros (5\%) e 4 amarelos $(1 \%)$. Duzentos e vinte pacientes $(72 \%)$ apresentaram forma clínica tipo remitente-recorrente. Oitenta e dois pacientes $(28 \%)$ apresentaram a forma progressiva $(50 \%$ forma secundária e $50 \%$ forma primariamente progressiva). Os sintomas iniciais mais comuns foram sensitivos $(31,7 \%)$ e ópticos $(26,8 \%)$.Em relação aos sintomas evolutivos predominavam os sintomas piramidais $(72,5 \%)$ e os medulares $(64,9 \%)$. A média do escore do EDSS final foi 3,37 e a do NRS foi 85,17. O índice anual de surtos foi 0,45. Nossos achados coincidem com os da literatura mundial, e ressaltam que 60 pacientes $(19,8 \%)$ apresentavam esclerose múltipla de forma benigna de valor prognóstico.
\end{abstract}

PALAVRAS-CHAVES: esclerose múltipla, estudo descritivo, formas evolutivas.

\section{Multiple sclerosis: descriptive study of the clinical forms in 302 cases}

ABSTRACT - Multiple sclerosis (MS) is one of the most common causes of chronic neurologic disability in young adults. We evaluate, through retrospective study, the epidemiological characteristics, the manifestations ways, the clinical manifestations, the evolution and the level of physical disability of MS in 302 patients. The average patients age was 37.7 and the relation between genders was 3.13F:1M. The average age at the beginning of MS was 29.6; 283 patients were white (94\%), 15 patients were black (5\%) and 4 patients were yellow; 220 patients $(72 \%)$ presented relapsing-remmiting clinical form; 82 patients $(28 \%)$ presented the progressive form (50\% secondary form and 50\% primarily progressive form). The most common initial symptoms were sensitive (31.7\%) and optical (26.8\%). The most common evolutive symptoms were pyramidal (72.5\%) and of the spinal cord (64.9\%). The average of the final EDSS was 3.37 and final NRS was 85.17. The index of the annual outbreak was 0.45 . Our findings coincide to the ones in the worldwide literature, however it is important to point out that 60 patients (19.8\%) presented benign MS of prognostic value.

KEY WORDS: multiple sclerosis, descriptive study, evolutive forms.

A esclerose múltipla (EM) é a desordem desmielinizante mais comum do sistema nervoso central (SNC), caracterizada por episódios repetidos de disfunção neurológica com remissão variável ${ }^{1}$.

Centro de Atendimento e Tratamento da Esclerose Múltipla (CATEM) de Clínica Neurológica da Faculdade de Ciências Médicas da Santa Casa de Misericórdia de São Paulo: *Neurologista; **Instrutor de Ensino; ***Professor-Adjunto. Aceite: 15 -fevereiro-2000.

Dr. Marcos A. Moreira - Rua Jaguaribe 619 / 6B - 01443-001 São Paulo/SP - Brasil. E-mail: neurologia@santacasasp.org.br 
A hipótese patogênica mais aceita é que a EM seja um fruto da conjunção de uma determinada predisposição genética e um fator ambiental desconhecido que ao se apresentarem num mesmo indivíduo, originariam uma disfunção do sistema imunológico, que desenvolveria uma ação autolesiva dirigida fundamentalmente contra a substância branca, com perda de oligodendrócitos e mielina, o que ocasionaria um defeito na condução dos impulsos nervosos e condicionaria o aparecimento dos sintomas. A predisposição genética, combinada ao fator ambiental desconhecido, estabelece ou mantém células T autorreativas que, após um período de latência de cerca de 10-20 anos, seriam ativadas por um fator sistêmico ou local (infecção viral, puerpério, etc). Uma vez ativadas, estas células T passam seletivamente pela barreira hemato-encefálica e ao serem expostas novamente ao auto-antígeno iniciam uma reação inflamatória (mediada por céls Th-1) ${ }^{2-5}$.

Estudos epidemiológicos têm permitido saber que a EM é a enfermidade neurológica crônica mais frequente em adultos jovens na Europa e América do Norte, e que a existência de um fator ambiental seria imprescindível para o aparecimento da doença, provavelmente na forma de uma infecção inaparente ou de caráter banal e, finalmente, estes estudos têm reconhecido a existência de um fator genético de susceptibilidade para a doença ${ }^{3,6}$. Apoiando o fator genético, Poser propôs uma hipótese segundo a qual a EM se produziria nas populações com antecedentes escandinavos, através das invasões vikings ${ }^{7}$.

No entanto, no Brasil, o que se tornou imprescindível é a interação dos vários centros especializados com a finalidade de realização de estudos multicêntricos que abordem os vários aspectos da doença. Além da dificuldade inerente ao diagnóstico, constituem-se em obstáculos para tal, a falta de colaboração de médicos no registro de casos e a precariedade da assistência médica estatal. Relatamos acerca de nossa experiência.

\section{MÉTODO}

Os dados referentes a 302 casos com diagnóstico de EM clinicamente definida (EMCDA1) segundo critérios estabelecidos por Poser e col. ${ }^{8}$ foram analisados através de protocolo específico (Tabela 1). A presente série foi obtida através do levantamento de prontuários na Disciplina de Neurologia da Faculdade de Ciências Médicas da Santa Casa de São Paulo e fichários de pacientes atendidos na clínica privada pelos médicos da equipe, no período de 1980 a 1997.

Tabela 1. Critérios diagnósticos para esclerose múltipla.

\begin{tabular}{|c|c|c|c|c|c|}
\hline Categoria & Surtos & Evidência Clínica & & Paraclínica & LCR BO/IgG \\
\hline \multicolumn{6}{|c|}{ A. Clinica/definida } \\
\hline EMCD A1 & 2 & 2 & & & \\
\hline EMCD A2 & 2 & 1 & $\underline{\mathrm{e}}$ & 1 & \\
\hline \multicolumn{6}{|c|}{ B. Laboratorial/ definida } \\
\hline EMLD B1 & 2 & 1 & $\underline{\mathrm{ou}}$ & 1 & + \\
\hline EMLD B2 & 1 & 2 & & & + \\
\hline EMLD B3 & 1 & 1 & $\underline{\mathrm{e}}$ & & + \\
\hline \multicolumn{6}{|c|}{ C. Clinica/ provável } \\
\hline EMCP C1 & 2 & 1 & & & \\
\hline EMCP C2 & 1 & 2 & & & \\
\hline EMCP C3 & 1 & 1 & $\underline{\mathrm{e}}$ & 1 & \\
\hline \multicolumn{6}{|c|}{ D. Laboratorial/ provável } \\
\hline EMLP D1 & 2 & & & & + \\
\hline
\end{tabular}

BO/IgG, bandas oligoclonais ou aumento de $\mathrm{IgG}$. 
Os critérios de seleção foram: pacientes portadores de EM formas remitente-recorrente (RR), primária (PP) e secundariamente progressivas (SP); tempo médio de doença superior a 8 anos; tempo médio de acompanhamento superior a 2,5 anos e realização do exame neurológico e aplicação das escalas de incapacidade funcional ${ }^{9,10}$ num intervalo de cerca de 6 meses. Além da definição clínica da doença, foi realizada ressonância magnética do crânio em 214 pacientes, exame do líquido cefalorraqueano (LCR) em 287 pacientes e exame dos potenciais evocados em 128 pacientes, como suporte laboratorial para o diagnóstico.

A forma de EM remitente-recorrente foi definida naqueles pacientes que apresentaram surtos claramente definidos com remissão completa ou incompleta; mantendo períodos sem progressão entre os surtos ${ }^{11}$. Surto (exacerbação,agudização) seria a ocorrência de sintomas de disfunção neurológica com mais de $24 \mathrm{~h}$ de duração incluindo dados de história de caráter subjetivo ou anamnéstico. Devem afetar diferentes partes do SNC e estar separados por um período mínimo de 1 mês ${ }^{8}$.

A forma de EM secundariamente progressiva foi definida naqueles pacientes que apresentavam um curso inicial tipo remitente-recorrente que posteriormente era sucedido por progressão com ou sem surtos ocasionais, discretas remissões, e platôs ${ }^{11}$.

A forma de EM primariamente progressiva foi definida naqueles pacientes que apresentavam curso progressivo desde o início com ocasionais platôs e/ou discretas flutuações ${ }^{11}$.

\section{RESULTADOS}

Os resultados constam das Tabelas 2 a 5.

A média da idade dos pacientes foi 37,7 anos e a relação entre os gêneros foi 3,13F:1M (229 mulheres e 73 homens). Em relação à raça , 283 pacientes eram brancos (94\%), 15 pacientes eram negros $(5 \%)$ e 4 eram amarelos (1\%). A média da idade ao início da EM foi 29,6 anos.

Duzentos e vinte pacientes $(72 \%)$ apresentaram forma clínica tipo remitente-recorrente. Oitenta e dois pacientes (28\%) apresentavam a forma progressiva (50\% forma secundária e $50 \%$ forma primariamente progressiva).

Os sintomas iniciais mais comuns foram: sensitivos $(31,7 \%)$,ópticos $(26,8 \%)$, piramidais $(17,8 \%)$, medulares $(16,5 \%)$, de tronco encefálico $(13,5 \%)$ e cerebelares $(5,9 \%)$. Os sintomas evolutivos (Tabela 3) caracterizavam-se por sintomas piramidais $(72,5 \%)$, medulares $(64,9 \%)$, sensitivos $(57,6 \%)$, ópticos $(53,9 \%)$, cerebelares $(33,4 \%)$ e de tronco encefalico $(31,7 \%)$. A média do Expanded Disability Status Scale (EDSS) inicial dos pacientes foi 3,23 e do EDSS final foi 3,37. A média do Neurologic Rating Scale (NRS) inicial dos pacientes foi 87,62 e do NRS final foi 85,17. $\mathrm{O}$ índice de anual de surtos foi 0,45 .

Tabela 2. Frequência (\%) dos sintomas iniciais nas diferentes formas em 302 pacientes com EM.

\begin{tabular}{lcccc}
\hline Sintomas e sinais & $\begin{array}{c}\mathrm{RR} \\
(\mathrm{n}=220)\end{array}$ & $\begin{array}{c}\mathrm{SP} \\
(\mathrm{n}=41)\end{array}$ & $\begin{array}{c}\mathrm{PP} \\
(\mathrm{n}=41)\end{array}$ & $\begin{array}{c}\text { Benigna } \\
(\mathrm{n}=60)\end{array}$ \\
\hline Piramidais & 11,3 & 17,0 & 12,1 & 15,0 \\
Medulares & 17,2 & 24,3 & 56,0 & 15,0 \\
Ópticos & 23,5 & 29,2 & 9,7 & 25,0 \\
Sensitivos & 29,5 & 14,6 & 2,4 & 20,0 \\
Tronco & 11,8 & 14,6 & 9,7 & 23,3 \\
Cerebelares & 4,0 & 9,7 & 12,1 & 3,3 \\
\hline
\end{tabular}


Tabela 3. Frequência (\%) dos sintomas evolutivos nas diferentes formas em 302 pacientes em EM.

\begin{tabular}{lcccc}
\hline Sinais e Sintomas & $\begin{array}{c}\mathrm{RR} \\
(\mathrm{n}=220)\end{array}$ & $\begin{array}{c}\mathrm{SP} \\
(\mathrm{n}=41)\end{array}$ & $\begin{array}{c}\mathrm{PP} \\
(\mathrm{n}=41)\end{array}$ & $\begin{array}{c}\text { Benigna } \\
(\mathrm{n}=60)\end{array}$ \\
\hline Piramidais & 60,0 & 58,5 & 68,2 & 68,3 \\
Medulares & 75,9 & 80,4 & 75,6 & 63,3 \\
Ópticos & 52,7 & 70,7 & 43,9 & 56,5 \\
Sensitivos & 56,3 & 60,9 & 60,9 & 48,3 \\
Tronco & 30,9 & 29,2 & 39,0 & 35,0 \\
Cerebelares & 29,5 & 39,0 & 48,7 & 31,6 \\
\hline
\end{tabular}

Tabela 4. Média de idade dos pacientes ao início da doença (anos) e relação homens /mulheres nas diferentes formas evolutivas.

\begin{tabular}{lcccc}
\hline Formas evolutivas & $\begin{array}{c}\mathrm{RR} \\
(\mathrm{n}=220)\end{array}$ & $\begin{array}{c}\mathrm{SP} \\
(\mathrm{n}=41)\end{array}$ & $\begin{array}{c}\mathrm{PP} \\
(\mathrm{n}=41)\end{array}$ & $\begin{array}{c}\text { Benigna } \\
(\mathrm{n}=60)\end{array}$ \\
\hline $\begin{array}{l}\text { Média da idade } \\
\text { ao início (anos) }\end{array}$ & 28,6 & 29,7 & 35,2 & 27,2 \\
Relação homens/mulheres & $1 / 3,5$ & $1 / 1,9$ & $1 / 2,7$ & $1 / 3,0$ \\
\hline
\end{tabular}

Tabela 5. Comparação entre a média do EDSS/NRS iniciais e finais dos pacientes nas diferentes formas clínicas.

\begin{tabular}{lcc}
\hline Formas clínicas & EDSS inicial/final & NRS inicial/final \\
\hline Remitente-recorrente $(\mathrm{n}=220)$ & $2,7 / 3,2$ & $89 / 88$ \\
Secundaria/ progressiva $(\mathrm{n}=41)$ & $4,3 / 4,9$ & $83 / 77$ \\
Primaria/ progressiva $(\mathrm{n}=41)$ & $4,5 / 5,9$ & $83 / 72$ \\
Benigna & $2,7 / 2,5$ & $88,5 / 88,8$ \\
\hline
\end{tabular}

\section{Forma remitente-recorrente}

Dos 302 casos de esclerose múltipla da presente série, 220 apresentavam forma remitenterecorrente $(72,8 \%)$. Destes, 172 pacientes $(78,1 \%)$ eram do gênero feminino e 48 do masculino. A média de idade dos pacientes foi 35,9 anos. A média da idade ao início da EM foi 28,6 anos.

Os sintomas iniciais mais comuns foram os sensitivos (29,5\%), ópticos $(23,6 \%)$, medulares $(17,2 \%)$ e piramidais $(11,3 \%)$. Os sintomas evolutivos caracterizavam-se por comprometimento medular (75,9\%), piramidal (60\%), sensitivo (56,3\%) e óptico $(52,7 \%)$. A média do EDSS inicial dos pacientes foi 2,7 e do final foi 3,2. O média do NRS inicial foi 89 e do final 88 . Foram totalizados 792 surtos e 277 surtos acompanhados (34,9\%). O índice anual de surtos foi 0,49/ano.

\section{Forma secundariamente progressiva}

Dos 302 casos, 41 apresentavam a forma secundariamente progressiva (13,6\%). Destes 27 pacientes $(65,8 \%)$ eram do gênero feminino e 14 do masculino. A média de idade dos pacientes foi 42,4 anos. A média da idade ao início da EM foi 29,7 anos. Os sintomas iniciais mais comuns foram ópticos 
$(29,2 \%)$, medulares $(24,3 \%)$, piramidais $(17 \%)$, sensitivos $(14,6 \%)$. Os sintomas evolutivos caracterizavam-se por comprometimento medular $(80,4 \%)$, ópticos $(70,7 \%)$, sensitivos $(60,9 \%)$, piramidal $(58,5 \%)$, cerebelar (39\%) e de tronco encefálico $(29,2 \%)$. A média do EDSS inicial dos pacientes foi 4,3 e do final foi 4,9. A média do NRS inicial foi 83 e do final 77. De um total aproximado de 157 surtos, acompanhamos 62 surtos. $\mathrm{O}$ índice anual de surtos destes pacientes foi 0,30/ano.

\section{Forma primariamente progressiva}

Dos 302 casos, 41 apresentavam a forma primariamente progressiva (13,6\%). Destes 30 pacientes eram do gênero feminino e 11 do masculino. A média de idade dos pacientes foi 42,8 anos. A média da idade ao início da EM foi 35,2 anos. Os sintomas iniciais mais comuns foram medulares $(56 \%)$, piramidais $(12,1 \%)$, cerebelares $(12,1 \%)$, óptico $(9,7 \%)$ e de tronco $(9,7 \%)$. Os sintomas evolutivos caracterizaram-se por comprometimento medular $(75,6 \%)$, piramidal $(68,2 \%)$, sensitivo $(60,9 \%)$, óptico $(43,9 \%)$, cerebelar $(48,7 \%)$ e de tronco encefálico (39\%). A média do EDSS inicial dos pacientes foi 4,5 e o final foi 5,9. A média do NRS inicial foi 83 e do final foi 72.

\section{Forma benigna}

Definida naqueles pacientes caracterizados como portadores da forma remitente-recorrente que apresentavam pelo menos 10 anos de história clínica e mantinham o EDSS menor ou igual $3^{12}$.

Dos 302 pacientes, 60 se enquadravam nestes critérios (19,8\%). Destes 45 pacientes eram do gênero feminino (75\%) e 15 do masculino (25\%). A média de idade dos pacientes foi 41,3 anos e ao início dos sintomas da doença foi 27,2 anos. Os sintomas iniciais mais comuns foram ópticos (25\%), de tronco $(23,3 \%)$, sensitivos $(20 \%)$, medulares $(15 \%)$, piramidais $(15 \%)$. Os sintomas evolutivos caracterizavam-se principalmente por comprometimento piramidal $(68,3 \%)$, medular $(63,3 \%)$, óptico $(56,5 \%)$, sensitivo $(48,3 \%)$, de tronco (35\%) e cerebelar (31,6\%). Do total de 267 surtos, 100 foram acompanhados pelos autores. O índice anual de surtos foi de 0,31. A média do EDSS inicial foi 2,7 e do final foi 2,5. A média do NRS inicial foi 88,5 e do final foi 88,8 .

\section{DISCUSSÃO}

Esta série merece críticas, sem dúvidas. A maioria dos casos foi analisada de modo retrospectivo. Este procedimento não nos permitiu determinar, em muitos casos, a evolução clínica de pacientes principalmente pelo curto período de acompanhamento. Por outro lado, é a maior série brasileira publicada e fornece alguns dados importantes, que contribuem para o maior conhecimento da doença no país.

Em relação à distribuição por raça, a EM é mais comum em brancos. Estudos epidemiológicos mostram baixa incidência entre negros e orientais e populações aparentemente resistentes a doença como os esquimós, maoris da Nova Zelândia, os lapões e os índios da América do Norte ${ }^{3,13}$. No Brasil, Papais-Alvarenga e col. encontraram $31,8 \%$ de pacientes negros num estudo realizado no Rio de Janeiro; fato este não corroborado pela nossa série (5\%) e pela de Callegaro e col. $(10 \%)^{14-16}$.

Martyn ${ }^{17}$ afirmou que o padrão de distribuição da doença por faixa etária não mudou. Continua rara antes da puberdade e após os 60 anos. Seu pico maior situa-se em torno de 30 anos $^{17}$. A média de idade de início dos sintomas de EM é semelhante em várias casuísticas. Em nossa série, a média de idade de início da doença foi 29,6 anos. Nos pacientes com a forma remitente-recorrente foi 28,6 anos; com a forma secundariamente progressiva foi 29,7 anos; e com a forma primariamente progressiva foi 35,2 anos. Leite e col. encontraram 31,4 anos, Lana e Lana 28,9 anos, sem diferenciar, entretanto, as formas remitentes das progressivas ${ }^{18-19}$.

Quanto à distribuição por gênero, a EM é mais comum nas mulheres. O predomínio da doença no gênero feminino é relatado por todos autores, havendo apenas diferenças de incidência de uma série para outra (Lana e Lana encontraram 1:2,3; Callegaro 1:1,6; Leite e col. 1:2,1) 13-16,18-21. $^{\text {. }}$

Cabe ressaltar alguns dados referentes aos pacientes com a forma primariamente progressiva. A comparação entre a média do EDSS final e inicial nas diferentes formas clínicas revelou valores maiores 
nesta forma. Além disso, predominaram os sintomas medulares como sintomas iniciais (56\%) e baixo comprometimento óptico $(9,7 \%)$. Na nossa série, o número de pacientes com esta forma representou $13,6 \%$ do total. No Brasil, Oliveira encontrou $30 \%$ de pacientes com esta forma clínica ${ }^{13}$. Papais-Alvarenga e cols encontraram apenas $4 \%{ }^{16}$. Em séries internacionais, Runnmarker e Andersen (22) reportaram $14 \%$ e Weinshenker e col. relataram $33 \%$ de formas primariamente progressivas ${ }^{23-25}$.

O índice anual de surtos, que correlaciona-se com o tempo de doença e o número de surtos, é variável segundo as séries, porém como média considera-se uma cifra de 0,85 /ano na fase remitente e de 0,30 se considerar todo o quadro evolutivo de pacientes. $\mathrm{O}$ intervalo entre os sintomas iniciais e o próximo surto é muito variável, um ano em $30 \%$, dois anos em $20 \%$, entre 5-9 anos em outros $20 \%$ e de 10-30 anos em $10 \%$ dos $\operatorname{casos}^{3,26}$. A nossa série mostra um índice anual de surtos na fase remitente de 0,49/ano e na forma secundariamente progressiva de 0,30/ano. Estes dados nos permitem inferir talvez, uma melhor evolução inicial dos nossos pacientes. No entanto, estudos prospectivos deverão ser realizados para melhor caracterização. Não encontramos dados publicados de séries brasileiras para fins de comparação.

Com relação ao comprometimento piramidal, os dois tipos mais comuns são: o cerebral (hemiparesia) e o medular. Arbitrariamente, resolvemos analisar e descrever separadamente o comprometimento medular nas diferentes formas clínicas. Definimos quadro medular nos casos caracterizados por para/quadriparesia, para/quadriparestesia e/ou disfunção esfincteriana. Obtivemos alguns dados importantes como a irrefutável predominância do quadro medular como sintoma inicial nos pacientes com a forma primariamente progressiva (56\%) e como sintoma evolutivo nos casos da forma secundariamente progressiva $(80,4 \%)$. Talvez o acometimento medular possa traduzir um "marcador" importante de cronicidade e pior prognóstico da doença.

McAlpine ${ }^{27}$ foi o primeiro a usar o termo EM benigna. Ele estudou 241 pacientes do Hospital Middlesex em Londres entre 1930 e 1949, todos com história clínica de início da doença dentro de 3 anos. Após cerca de 10 anos de observação, McAlpine relatou em 1959 que 32\% mantinham-se estáveis. Em 1964, decorridos 5 anos, contudo, $15 \%$ destes pacientes aumentaram a sua incapacidade ${ }^{28}$.

Entretanto, foi Thompson ${ }^{12}$ em 1986, quem definiu como critério de EM benigna o escore do EDSS menor ou igual a 3 (consegue deambular sem nenhuma ajuda) após 10 anos de evolução da doença $^{12}$. Em nossa série encontramos $19,8 \%$ dos pacientes que preencheram estes critérios. Um dado interessante foi que predominou o comprometimento óptico como sintoma inicial (25\%), seguido do tronco encefálico $(23,3 \%)$ e sensitivo (20\%). Como sintomas evolutivos destacam-se os piramidais $(68,3 \%)$, medulares e ópticos. Em relação a séries brasileiras este fato foi abordado somente por Oliveira ${ }^{13}$ em sua série de 50 pacientes na qual evidenciou-se $8 \%$ dos pacientes. Deixamos claro que EM benigna não é um diagnóstico, mas um prognóstico e deve ser analisada em termos de relativa probabilidade. Nem sempre é significado de bom prognóstico, pois mesmo os pacientes que preenchem estes critérios podem vir a desenvolver certo grau de incapacidade ${ }^{3,26,29}$.

A forma surto-progressiva não foi abordada principalmente devido ao fato de ter sido recentemente descrita. Segundo Lublin e Reingold ${ }^{11}$ é a forma mais difícil de ser definida, e caracterizase como doença progressiva desde o início, com surtos claramente definidos com ou sem remissão completa sendo os períodos entre os surtos caracterizados por contínua progressão. Sabemos que as formas progressivas carregam consigo valor preditivo maior para uma evolução grave. Estudos prospectivos deverão ser realizados para estimar mais objetivamente o valor prognóstico e terapêutico dos pacientes que evoluem com a forma surto-progressiva.

Na ausência de achados clínicos patognomônicos ou testes laboratoriais definitivos, a esclerose múltipla ainda permanece um diagnóstico de exclusão. No entanto, uma história clínica bem realizada apoiada em métodos de investigação paraclínicos (LCR, potenciais evocados, ressonância magnética) permitem descartar com bastante segurança outras enfermidades e chegar a uma definição diagnóstica e cada vez mais precoce da EM na maioria dos casos.

Atualmente, com a introdução das novas estratégias de tratamento baseadas na imunomodulação, a caracterização precisa das formas evolutivas da enfermidade tornou-se imperativa para a correta indicação do tratamento a ser conduzido. 


\section{REFERÊNCIAS}

1. Kurtzke JF, Beebe GW, Dagler B, et al. Studies on the natural history of multiple sclerosis: clinical and laboratory findings at first diagnosis. Acta Neurol Scand 1972;48:19-46.

2. Compston A. Esclerosis multiple. In Brostoff J, Scadding GK, Male DK, Roitt IM (eds). Inmunología clínica. London: Gower Med Publ 1994:9.2-9.7.

3. Fernández O, Fernández VE. Esclerosis múltiple: una enfermedad relativamente frecuente en España. Fundación Española de Esclerosis Múltiple (FEDEM).Málaga,1997.

4. Hartung HP, Archelos JJ, Zielasek J, et al. Circulating adhesion molecule and inflammatory mediators in demyelination: a review. Neurology 1995;45(S6):S22-S32.

5. Hillert J. Human leukocyte antigen studies in multiple sclerosis. Ann Neurol 1994;36:S15-S17

6. Sadovnick AD. Genetic epidemiology of multiple sclerosis: a survey. Ann Neurol 1994;36(S2):S194-S203.

7. Poser CM. Viking voyages: the origin of multiple sclerosis? An essay in medical history. Acta Neurol Scand 1995;161:11-22.

8. Poser CM, Paty DW,Scheinberg L,et al. New diagnostic criteria for multiple sclerosis: guidelines for research protocols. Ann Neurol 1983;13:227-231.

9. Kurtzke JF. Rating neurologic impairment in multiple sclerosis: an expanded disability status scale (EDSS). Neurology 1983;33:1444-52.

10. Sipe JC, Knobler RL, Braheny SL, et al. A neurologic rating scale (NRS) for use in multiple sclerosis. Neurology 1984;34:1368-1372.

11. Lublin FD, Reingold SC. Defining the clinical course of multiple sclerosis. Neurology 1996;46:907-911.

12. Thompson AJ, Hutchinson M, Brazil J, et al. A clinical and laboratory study of benign multiple sclerosis. Q J Med 1986;58:69-80.

13. Oliveira EML. Estudo retrospectivo de 50 pacientes do ambulatório de doenças neuromusculares da UNIFESP - EPM, no período de 1983-1995. Tese de Mestrado, Escola Paulista de Medicina UNIFESP. São Paulo, 1997.

14. Callegaro D. Contribuição do estudo clínico evolutivo da esclerose múltipla: análise de 120 pacientes. Tese de Doutorado, Faculdade de Medicina da Universidade de São Paulo. São Paulo, 1989.

15. Callegaro D, Lolio CA, Radvany J, Tilbery CP, Mendonça RA, Melo ACP. Prevalence of multiple sclerosis in the city of São Paulo, Brazil in 1990. Neuroepidemiology 1992;11:11-14.

16. Papais-Alvarenga RM, Santos CMM, Bernadini JSA, Camargo SMGG. Esclerose múltipla:perfil clínico e epidemiológico no Rio de Janeiro. Arq Neuropsiquiatr 1990;48(Supl):63A.

17. Martyn C. The epidemiology of multiple sclerosis. In Matthews WB, Compston A, Allen IV, Martyn CD (eds). McAlpine's multiple sclerosis. 2.ed. New York: Churchill Livingstone, 1991:3-42.

18. Lana-Peixoto MA, Lana-Peixoto MI. Is multiple sclerosis in Brazil and Asia alike? Arq Neuropsiquiatr 1992;50:419-425.

19. Leite ACCB, Andrade C, Novis S. Esclerose múltipla no Rio de Janeiro: apresentação clínica em 51 casos. Arq Neuropsiquiatr 1990;48 (Supl):66 ${ }^{\mathrm{A}}$.

20. Rolak LA. The diagnosis of Multiple sclerosis. Neurol Clinic 1996;14:27-42.

21. Tilbery CP, Felipe E, Baldauf CM, Peres MFP. Esclerose múltipla: análise clínica e evolutiva de 214 casos. Arq Neuropsiquiatr 1995;53:203-207.

22. Runmarker B, Andersen O. Prognostic factors in a multiple sclerosis incidence cohort with twenty-five years of follow-up. Brain 1993;116:117-134.

23. Weinshenker BG, Bass B, Rice GPA, et al. The natural history of multiple sclerosis: a geographically based study. 1 . Clinical course and disability. Brain 1989;112:133-146.

24. Weinshenker BG, Bass B, Rice GPA, et al. The natural history of multiple sclerosis: a geographically based study. 2. Predictive value of the early clinical course. Brain 1989;112:1419-1428.

25. Weinshenker BG, Bass B, Rice GPA, et al. The natural history of multiple sclerosis: a geographically based study. 3 . Multivariate analysis of predictive factors and models of outcome. Brain 1991;114:1045-1056.

26. Mattews WB. Clinical aspects. In Matthews WB, Compston A, Allen IV, Martyn CN (eds). Mc Alpine's multiple sclerosis. $2^{\mathrm{a}}$.ed. Edinburgh: Churchill Livingstone, 1991:P231-250.

27. McAlpine D. The benign form of multiple sclerosis: a study based on 241 cases seen within 3 years of onset and followed up until the 10h year or more of the disease. Brain 1961;84:186-203.

28. Mc Alpine D. The benign form of multiple sclerosis: results of a long-term study. Br Med J 1964;2:1029-1032.

29. Weinshenker BG. Epidemiology of multiple sclerosis. Neurol Clin 1995;14:291-308. 